Portland State University

PDXScholar

$10-22-2010$

\title{
Accurate description of the optical response of a multilayered spherical system in the long wavelength approximation
}

\author{
H. Y. Chung \\ G. Y. Guo \\ Hai-Pang Chiang \\ D. P. Tsai \\ P.T. Leung \\ Portand State University
}

Follow this and additional works at: https://pdxscholar.library.pdx.edu/phy_fac

Part of the Physics Commons

Let us know how access to this document benefits you.

\section{Citation Details}

Chung, H. Y.,Guo, G. Y., Chiang, H. P., Tsai, D. P., \& Leung, P. T. (2010). Accurate description of the optical response of a multilayered spherical system in the long wavelength approximation. Physical Review B, $82(16)$

This Article is brought to you for free and open access. It has been accepted for inclusion in Physics Faculty Publications and Presentations by an authorized administrator of PDXScholar. Please contact us if we can make this document more accessible: pdxscholar@pdx.edu. 


\title{
Accurate description of the optical response of a multilayered spherical system in the long wavelength approximation
}

\author{
H. Y. Chung (鍾弘毅), ${ }^{1, *, \dagger}$ G. Y. Guo (郭光宇), ${ }^{1,2}$ H.-P. Chiang (江海邦), ${ }^{3,4,5}$ \\ D. P. Tsai (蔡定平), ${ }^{1,5}$ and P. T. Leung (梁培德) $)^{1,3,6, *, \$}$ \\ ${ }^{1}$ Department of Physics, National Taiwan University, Taiwan 10617, Republic of China \\ ${ }^{2}$ Graduate Institute of Applied Physics, National Chengchi University, Taiwan 11605, Republic of China \\ ${ }^{3}$ Institute of Optoelectronic Sciences, National Taiwan Ocean University, Keelung, Taiwan 202, Republic of China \\ ${ }^{4}$ Institute of Physics, Academia Sinica, Taipei, Taiwan 11529, Republic of China \\ ${ }^{5}$ Instrument Technology Research Center, National Applied Research Laboratories, Hsinchu, Taiwan 300, Republic of China \\ ${ }^{6}$ Department of Physics, Portland State University, P.O. Box 751, Portland, Oregon 97207-0751, USA
}

(Received 26 August 2010; revised manuscript received 27 September 2010; published 22 October 2010; corrected 27 October 2010)

\begin{abstract}
The optical response of a multilayered spherical system of unlimited number of layers (a "matryoshka") in the long wavelength limit can be accounted for from the knowledge of the static multipole polarizability of the system to first-order accuracy. However, for systems of ultrasmall dimensions or systems with sizes not-toosmall compared to the wavelength, this ordinary quasistatic long wavelength approximation (LWA) becomes inaccurate. Here we introduce two significant modifications of the LWA for such a nanomatryoshka in each of the two limits: the nonlocal optical response for ultrasmall systems $(<10 \mathrm{~nm})$, and the "finite-wavelength corrections" for systems $\sim 100 \mathrm{~nm}$. This is accomplished by employing the previous work for a single-layer shell, in combination with a certain effective-medium approach formulated recently in the literature. Numerical calculations for the extinction cross sections for such a system of different dimensions are provided as illustrations for these effects. This formulation thus provides significant improvements on the ordinary LWA, yielding enough accuracy for the description of the optical response of these nanoshell systems over an appreciable range of sizes, without resorting to more involved quantum mechanical or fully electrodynamic calculations.
\end{abstract}

DOI: 10.1103/PhysRevB.82.165440

PACS number(s): 78.67.Pt, 73.20.Mf, 73.21.Ac, 41.20.Cv

\section{INTRODUCTION}

The recent surge in the study of plasmonics has opened up the possibility for an ultimate unification of nanophotonics with nanoelectronics in future device design. ${ }^{1}$ Among the various nanoplasmonic systems, metallic nanoparticles (MNPs) have become a very promising one for their relatively easy manufacturing and manipulation in many significant applications, such as in spectroscopic enhancement ${ }^{2}$ and fabrication of nanowaveguides. ${ }^{3}$ Hence a thorough understanding of the optical properties of these MNPs is of great significance in the study of nanoplasmonics. ${ }^{4}$

It is well known that for a given MNP of known material composition (i.e., the kind of metal and dielectric material in the system), the geometrical configuration is the most important factor for the control of the optical properties of the MNP. Of the many configurations such as spherical, spheroidal, pyramidal, etc., it has been known for more than a decade that the shell configuration is one of the most versatile systems in its tunability of the plasmon resonances. First fabricated in $1998,{ }^{5}$ these metallic nanoshells have received great attention from researchers of various fields due partly to their unique applications in especially the biomedical areas. ${ }^{6}$ Significant developments in these nanoshells have taken place in the last decade including the fabrication of the spheroidal shells $\left(\right.$ nanorice ${ }^{7}$ ), bimetallic shells, ${ }^{8}$ and the "nanomatryoshka." "9 This last system refers to a multilayered structure consisting of various metallic and dielectric core/ layers which will enable even greater tunability in the plasmonic resonances of the nanoshell.
Besides developments in fabrication/experimental studies, significant theories and models have also been advanced for the understanding of the intriguing optical properties of these nanoshells. For example, a general transfer-matrix method has been applied to formulate the description of optical interaction with the nanomatryoshka ${ }^{10}$ and in the long wavelength limit, very successful theories have been established for the description of the coupled plasmonic modes and the optical response through the polarizability of these nanoshells, including the hybridization model ${ }^{9,11}$ and the effective-medium approach. ${ }^{12,13}$ However, while these quasistatic theories in the long wavelength approximation (LWA) are powerful and much simpler than those from a fully electrodynamic formulation, they suffer inaccuracies in a significant way when the size of the system becomes either ultrasmall $(<10 \mathrm{~nm})$ or not-too-small compared to the optical wavelengths (say, $\sim 100 \mathrm{~nm}$ ).

In the ultrasmall limit, nontrivial microscopic optical effects will emerge when the quantum nature of the electrons in the metal can no longer be ignored. Effects such as those due to quantum confinement and the spilling of the electrons over the geometric boundary, for example, will become significant when the size of a MNP shrinks to below $\sim 10 \mathrm{~nm}$. While accounting for these subtle quantum effects in nanoplasmonics is by no means straightforward, and fully quantum mechanical calculations for real metallic nanostructures (e.g., using time-dependent density-functional theories ${ }^{14}$ ) are limited to small sizes and are highly computational as the number of electrons increases, a compromising approach 


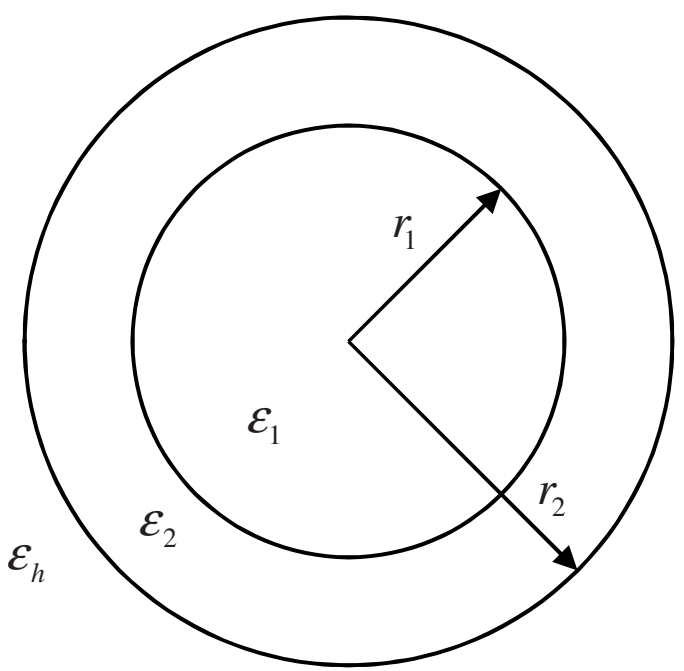

FIG. 1. Configuration of a single-shell system.

will be the formulation using nonlocal optics with partial quantum effects introduced through the nonlocal dielectric function of the metals. ${ }^{15}$

In fact, nonlocal electrodynamics for systems of small dimensions (e.g., thin films, small particles, etc.) has been actively studied for over several decades, and one of the exciting recent developments has been in the works on nonlocal numerical electrodynamics which enables treatments of MNPs of irregular geometry. ${ }^{16-18}$ For systems of spherical symmetry, both nonlocal electrodynamic transfer matrices ${ }^{10}$ and nonlocal electrostatic polarizabilities ${ }^{19-21}$ have been established in the literature. While the formulation in Ref. 10 is rather general and can be applied to a nanomatryoshka via rather complicated mathematical manipulations, the previous simpler results for the polarizabilities ${ }^{20,21}$ were largely focused on a single-layered shell system. Although the approach using "impedance transfer relations" in Ref. 20 can be generalized to a system of large number of shells, the iteration scheme and detailed studies have not been carried out except for a single-shell structure.

In the limit when the dimension of the multishell system gets to $\sim 100 \mathrm{~nm}$, the nonlocal effects can be ignored but there will be significant corrections due to the finiteness of the optical wavelength. One rather successful approach to account for such corrections is known as the modified LWA (MLWA), which has been established in the literature for particles ${ }^{4,22,23}$ and simple shell structures of these dimensions. ${ }^{13}$ Although this MLWA is limited only to the modification of the dipolar response of the system, it has been checked for consistency with the lowest-order result from the exact Mie theory for a spherical particle, ${ }^{22,23}$ and has been proven to be sufficiently accurate when compared to exact calculations for single spherical particles up to sizes of $\sim 100 \mathrm{~nm}^{24}$

It is the purpose of our present work to provide a formulation of both the above two modifications (i.e., the nonlocal effects and the MLWA) in the two limits of multishell size in a unified approach, based on an effective-medium approach introduced recently in the literature. ${ }^{12,13}$ We shall first reformulate the previous results ${ }^{20,21}$ for the case of a nonlocal nanomatryoshka composed of an unlimited number of metallic/dielectric shells adopting a slightly different approach. Our method is a combination of the result for a single shell ${ }^{20}$ with the effective-medium approach which we recently established ${ }^{13}$ based on the generalization of earlier works. ${ }^{12}$ Next we shall use the same approach to introduce electrodynamic corrections for multishells of dimensions $\sim 100 \mathrm{~nm}$ in the MLWA limited only to local response theory since nonlocal effects are insignificant for these relatively large systems. We shall present our model in Sec. II, followed with numerical illustration via calculation of the field intensity and extinction coefficients of these MNPs in Sec. III, and conclusion with a discussion on the limitation and potential applications of our results.

\section{THEORETICAL MODEL}

\section{A. Nonlocal polarizability for a nanomatryoshka}

The nonlocal multipole polarizability $\left(\alpha_{\ell}\right)$ for a spherical system has been derived in the literature adopting the semiclassical infinite barrier approximation for a solid sphere ${ }^{19}$ and spherical shells (or coated spheres), ${ }^{20,21}$ respectively. Here we provide an alternative formulation for a multishell system of unlimited number of layers using an effectivemedium approach. ${ }^{12,13}$ We shall see that an effective nonlocal dielectric function can be clearly introduced in this approach. To introduce our present notations and to explain our ideas of generalizing the results to the nanomatryoshka, we first recapitulate the results in Ref. 20 in slightly different notations as follows. With reference to the single-layered shell in Fig. 1, the previous result ${ }^{20,21}$ can be expressed as follows:

$$
\alpha_{\ell}=r_{2}^{2 \ell+1} \frac{b_{2}\left(1-\varepsilon_{h} / a_{2}\right)\left[\ell \xi_{1}+(\ell+1) c_{2}\right] r_{2}^{2 \ell+1}+c_{2}\left[(\ell+1)\left(\xi_{1}-b_{2}\right)+\ell \varepsilon_{h}\left(\xi_{1} / b_{2}-1\right)\right] r_{1}^{2 \ell+1}}{b_{2}\left[1+(\ell+1) \varepsilon_{h} / \ell a_{2}\right]\left[\ell \xi_{1}+(\ell+1) c_{2}\right] r_{2}^{2 \ell+1}+c_{2}(\ell+1)\left[\varepsilon_{h}-b_{2}+\xi_{1}\left(1-\varepsilon_{h} / b_{2}\right)\right] r_{1}^{2 \ell+1}},
$$

where $\varepsilon_{h}$ is the local and real dielectric constant of the hosting medium outside the shell, and the various coefficients are defined as $a_{n}=E_{\ell, n}^{n, n}, b_{n}=E_{\ell, n}^{n-1, n}$, and $c_{n}=E_{\ell, n}^{n-1, n-1}$ with 


$$
E_{\ell, n}^{p, q}=\frac{\pi}{2(2 \ell+1)} \frac{r_{p}^{\ell}}{r_{q}^{\ell+1}}\left[\int_{0}^{\infty} \frac{j_{\ell}\left(k r_{p}\right) j_{\ell}\left(k r_{q}\right)}{\varepsilon_{n}(k, \omega)} d k\right]^{-1},
$$

and $\xi_{1}=a_{1}$ for this simple layered structure. Note that the integrand in Eq. (2) is expressed in terms of the spherical Bessel functions as well as the nonlocal dielectric function of the $n$th layer, ${ }^{20}$ with $n=1$ referring to just the core. Note also that in the case of local dielectric response, $\varepsilon_{n}(k, \omega)$ $\rightarrow \varepsilon_{n}(\omega)$ and we have

$$
E_{\ell, n}^{p, q} \rightarrow \varepsilon_{n}(\omega) .
$$

To generalize the above results to the nanomatryoshka system which contains a large number of $(n+1)$ shells [Fig. 2(a)], we follow the effective-medium idea of previous works ${ }^{12,13}$ to introduce an effective nonlocal function $\varepsilon_{n}^{s}(k, \omega)$ which will represent the nonlocal response of all the shells contained in the radii $r \leq r_{n}$ [Fig. 2(b)]. We shall see that this will introduce the following equivalent local response function:

$$
\xi_{n}=\frac{\pi}{2(2 \ell+1)} \frac{1}{r_{n}}\left[\int_{0}^{\infty} \frac{j_{\ell}\left(k r_{n}\right) j_{\ell}\left(k r_{n}\right)}{\varepsilon_{n}^{s}(k, \omega)} d k\right]^{-1} .
$$

Note that $\xi_{n} \neq a_{n}$ except for $n=1$ since $\varepsilon_{n}^{s}(k, \omega)=\varepsilon_{n}(k, \omega)$ only for $n=1$. To determine $\varepsilon_{n}^{s}(k, \omega)$ (or equivalently the corresponding $\xi_{n}$ ), we consider the situation when the two outermost layers become identical media (i.e., $\varepsilon_{n+1}=\varepsilon_{n}$ ), in
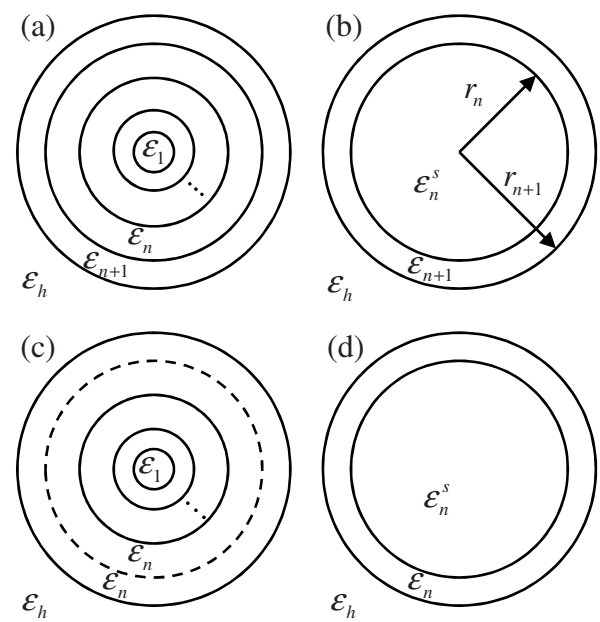

FIG. 2. Illustration of the approach using the effective-medium idea. See text for details.

which case the original system [Fig. 2(a)] and the effective system [Fig. 2(b)] assume the configurations as depicted in Figs. 2(c) and 2(d), respectively. Thus, the result in Eq. (1) can now be applied to Fig. 2(c) with $r_{1} \rightarrow r_{n-1}, r_{2} \rightarrow r_{n+1}, \varepsilon_{1}$ $\rightarrow \varepsilon_{n-1}^{s}, \varepsilon_{2} \rightarrow \varepsilon_{n}$ and similarly to Fig. 2(d) with $r_{1} \rightarrow r_{n}, r_{2}$ $\rightarrow r_{n+1}, \varepsilon_{1} \rightarrow \varepsilon_{n}^{s}, \varepsilon_{2} \rightarrow \varepsilon_{n}$, respectively. By using the above two replacements into Eq. (1) and equating the two resultant expressions for the polarizabilities, one has

$$
\begin{gathered}
\frac{f_{n}\left(1-\varepsilon_{h} / e_{n}\right)\left[\ell \xi_{n-1}+(\ell+1) c_{n}\right] r_{n+1}^{2 \ell+1}+c_{n}\left[(\ell+1)\left(\xi_{n-1}-f_{n}\right)+\ell \varepsilon_{h}\left(\xi_{n-1} / f_{n}-1\right)\right] r_{n-1}^{2 \ell+1}}{f_{n}\left[1+(\ell+1) \varepsilon_{h} / \ell e_{n}\right]\left[\ell \xi_{n-1}+(\ell+1) c_{n}\right] r_{n+1}^{2 \ell+1}+c_{n}(\ell+1)\left[\varepsilon_{h}-f_{n}+\xi_{n-1}\left(1-\varepsilon_{h} / f_{n}\right)\right] r_{n-1}^{2 \ell+1}} \\
\quad=\frac{d_{n}\left(1-\varepsilon_{h} / e_{n}\right)\left[\ell \xi_{n}+(\ell+1) a_{n}\right] r_{n+1}^{2 \ell+1}+a_{n}\left[(\ell+1)\left(\xi_{n}-d_{n}\right)+\ell \varepsilon_{h}\left(\xi_{n} / d_{n}-1\right)\right] r_{n}^{2 \ell+1}}{d_{n}\left[1+(\ell+1) \varepsilon_{h} / \ell e_{n}\right]\left[\ell \xi_{n}+(\ell+1) a_{n}\right] r_{n+1}^{2 \ell+1}+a_{n}(\ell+1)\left[\varepsilon_{h}-d_{n}+\xi_{n}\left(1-\varepsilon_{h} / d_{n}\right)\right] r_{n}^{2 \ell+1}},
\end{gathered}
$$

where $d_{n}=E_{\ell, n}^{n, n+1}, e_{n}=E_{\ell, n}^{n+1, n+1}$, and $f_{n}=E_{\ell, n}^{n-1, n+1}$.

Now define the left-hand side of Eq. (5) as

$$
F_{n}=\frac{f_{n}\left(1-\varepsilon_{h} / e_{n}\right)\left[\ell \xi_{n-1}+(\ell+1) c_{n}\right] r_{n+1}^{2 \ell+1}+c_{n}\left[(\ell+1)\left(\xi_{n-1}-f_{n}\right)+\ell \varepsilon_{h}\left(\xi_{n-1} / f_{n}-1\right)\right] r_{n-1}^{2 \ell+1}}{f_{n}\left[1+(\ell+1) \varepsilon_{h} / \ell e_{n}\right]\left[\ell \xi_{n-1}+(\ell+1) c_{n}\right] r_{n+1}^{2 \ell+1}+c_{n}(\ell+1)\left[\varepsilon_{h}-f_{n}+\xi_{n-1}\left(1-\varepsilon_{h} / f_{n}\right)\right] r_{n-1}^{2 \ell+1}} .
$$

Note that $F_{n}$ is a function of $\xi_{n-1}$. Then a relation between $\xi_{n}$ and $\xi_{n-1}$ can be obtained in the following form:

$$
\xi_{n}=\frac{d_{n}-(1+1 / \ell) a_{n} q_{n}}{1+q_{n}}
$$

where

$$
q_{n}=\frac{e_{n} \ell\left(1-F_{n}\right)-\varepsilon_{h}\left[\ell+(\ell+1) F_{n}\right]}{d_{n}(\ell+1)\left(1-F_{n}\right)+\varepsilon_{h}\left[\ell+(\ell+1) F_{n}\right]} \frac{d_{n}^{2}}{a_{n} e_{n}}\left(\frac{r_{n+1}}{r_{n}}\right)^{2 \ell+1} .
$$

Equations (6)-(8) then provide an implicit iterative scheme for the calculation of $\xi_{n}$ in terms of ultimately $\xi_{1}=a_{1}$ which can be calculated from Eq. (2) once the nonlocal dielectric function of the core is known. Once $\xi_{n}$ is known, the nonlocal polarizability of the whole $(n+1)$-shell system can then be obtained using an equation similar to Eq. (1) and the final result can be expressed systematically by introducing another function as follows:

$$
G_{n}=\frac{b_{n}\left(1-\varepsilon_{h} / a_{n}\right)\left[\ell \xi_{n-1}+(\ell+1) c_{n}\right] r_{n}^{2 \ell+1}+c_{n}\left[(\ell+1)\left(\xi_{n-1}-b_{n}\right)+\ell \varepsilon_{h}\left(\xi_{n-1} / b_{n}-1\right)\right] r_{n-1}^{2 \ell+1}}{b_{n}\left[1+(\ell+1) \varepsilon_{h} / \ell a_{n}\right]\left[\ell \xi_{n-1}+(\ell+1) c_{n}\right] r_{n}^{2 \ell+1}+c_{n}(\ell+1)\left[\varepsilon_{h}-b_{n}+\xi_{n-1}\left(1-\varepsilon_{h} / b_{n}\right)\right] r_{n-1}^{2 \ell+1}} .
$$

The polarizability for the whole system can then be obtained in the form 


$$
\alpha_{\ell}=r_{n+1}^{2 \ell+1} G_{n+1} .
$$

Note that this result can also be obtained by iterating the impedance transfer relations as derived in Ref. 20. It is easy to check that Eqs. (9) and (10) indeed lead back to the correct results in the limit for simple systems. For example, for a single solid sphere, setting $n=0$ in Eqs. (9) and (10) leads to

$$
\alpha_{\ell}=r_{1}^{2 \ell+1} G_{1}=r_{1}^{2 \ell+1} \frac{1-\varepsilon_{h} / a_{1}}{1+(\ell+1) \varepsilon_{h} / \ell a_{1}} .
$$

When the result in Eq. (2) is used for $a_{1}$, Eq. (11) reproduces a result first derived in Ref. 19. For a single-layered shell (i.e., a coated sphere), setting $n=1$ in Eqs. (9) and (10) leads to

$$
\alpha_{\ell}=r_{2}^{2 \ell+1} G_{2}=r_{2}^{2 \ell+1} \frac{b_{2}\left(1-\varepsilon_{h} / a_{2}\right)\left[\ell \xi_{1}+(\ell+1) c_{2}\right] r_{2}^{2 \ell+1}+c_{2}\left[(\ell+1)\left(\xi_{1}-b_{2}\right)+\ell \varepsilon_{h}\left(\xi_{1} / b_{2}-1\right)\right] r_{1}^{2 \ell+1}}{b_{2}\left[1+(\ell+1) \varepsilon_{h} / \ell a_{2}\right]\left[\ell \xi_{1}+(\ell+1) c_{2}\right] r_{2}^{2 \ell+1}+c_{2}(\ell+1)\left[\varepsilon_{h}-b_{2}+\xi_{1}\left(1-\varepsilon_{h} / b_{2}\right)\right] r_{1}^{2 \ell+1}}
$$

a result identical to Eq. (1). In addition, in the case when all the shells are described by some local dielectric functions, then we have

$$
a_{n}=b_{n}=c_{n}=d_{n}=e_{n}=f_{n}=\varepsilon_{n}(\omega) \text { and } \xi_{n}=\varepsilon_{n}^{s}(\omega),
$$

and Eqs. (6)-(10) then provide an iterative scheme for the computation of the polarizability of the nanomatryoshka, identifying with that established in our previous work. ${ }^{13}$

\section{B. MLWA for a nanomatryoshka}

In the other limit with the shell dimensions $\gg 10 \mathrm{~nm}$ 's, nonlocal effects become unimportant but the finiteness of the wavelengths can no longer be ignored. Here we follow our previous work ${ }^{13}$ to introduce these "finite-wavelength corrections" to the polarizability of a nanomatryoshka according to the MLWA, limited only to the local dielectric response of the system.

The main idea of the original MLWA (Ref. 22) is to modify the electrostatic depolarization field inside a polarizable sphere, by including (i) the dynamic depolarization due to retardation and (ii) the radiation damping field acting on the induced dipoles in the sphere. Such modifications are hence limited only to the dipole response of the sphere. ${ }^{4,22,23}$ While a straightforward generalization of the method in Ref. 22 to a multishell structure is not trivial, the application of the effective-medium idea makes this generalization possible by reducing the multishell system to an effective sphere. ${ }^{12,13}$ For a single-layered shell (Fig. 1), we have obtained previously the following MLWA dipole polarizability: ${ }^{13}$

$$
\alpha_{1 \mathrm{ML}}=\frac{1}{3} r_{2}^{3} \frac{\left(\varepsilon_{2}-\varepsilon_{h}\right)\left[\varepsilon_{1} \chi_{1}+\varepsilon_{2}\left(1-\chi_{1}\right)\right] r_{2}^{3}+\left(\varepsilon_{1}-\varepsilon_{2}\right)\left[\varepsilon_{2}\left(1-\chi_{2}\right)+\varepsilon_{h} \chi_{2}\right] r_{1}^{3}}{\left[\varepsilon_{1} \chi_{1}+\varepsilon_{2}\left(1-\chi_{1}\right)\right]\left[\varepsilon_{2} \chi_{2}+\varepsilon_{h}\left(1-\chi_{2}\right)\right] r_{2}^{3}+\left(\varepsilon_{1}-\varepsilon_{2}\right)\left(\varepsilon_{2}-\varepsilon_{h}\right) \chi_{2}\left(1-\chi_{2}\right) r_{1}^{3}},
$$

where

$$
\chi_{j}=\frac{1}{3}-\frac{1}{3} y_{j}^{2}-i \frac{2}{9} y_{j}^{3}
$$

with $y_{j}=k r_{j}=2 \pi r_{j} / \lambda$, and $r_{1}$ and $r_{2}$ are the radius of the inner and outer surfaces, respectively.

In the limit $\lambda \rightarrow \infty$, Eq. (14) leads back to the simple local result for the dipole polarizability of a single-layered shell,

$$
\alpha_{1}=r_{2}^{3} \frac{\left(\varepsilon_{2}-\varepsilon_{h}\right)\left(\varepsilon_{1}+2 \varepsilon_{2}\right) r_{2}^{3}+\left(\varepsilon_{1}-\varepsilon_{2}\right)\left(2 \varepsilon_{2}+\varepsilon_{h}\right) r_{1}^{3}}{\left(\varepsilon_{1}+2 \varepsilon_{2}\right)\left(\varepsilon_{2}+2 \varepsilon_{h}\right) r_{2}^{3}+2\left(\varepsilon_{1}-\varepsilon_{2}\right)\left(\varepsilon_{2}-\varepsilon_{h}\right) r_{1}^{3}} .
$$

To generalize the above results for the nanomatryoshka, we start with Eq. (5), setting $\ell=1$ and applying the conditions in Eq.

(13) for the local response case leads to the following result:

$$
\frac{\left(1-\varepsilon_{h} / \varepsilon_{n}\right)\left[\varepsilon_{n-1}^{s}+2 \varepsilon_{n}\right] r_{n+1}^{3}+\left[2\left(\varepsilon_{n-1}^{s}-\varepsilon_{n}\right)+\varepsilon_{h}\left(\varepsilon_{n-1}^{s} / \varepsilon_{n}-1\right)\right] r_{n-1}^{3}}{\left[1+2 \varepsilon_{h} / \varepsilon_{n}\right]\left[\varepsilon_{n-1}^{s}+2 \varepsilon_{n}\right] r_{n+1}^{3}+2\left[\varepsilon_{h}-\varepsilon_{n}+\varepsilon_{n-1}^{s}\left(1-\varepsilon_{h} / \varepsilon_{n}\right)\right] r_{n-1}^{3}}=\frac{\left(1-\varepsilon_{h} / \varepsilon_{n}\right)\left[\varepsilon_{n}^{s}+2 \varepsilon_{n}\right] r_{n+1}^{3}+\left[2\left(\varepsilon_{n}^{s}-\varepsilon_{n}\right)+\varepsilon_{h}\left(\varepsilon_{n}^{s} / \varepsilon_{n}-1\right)\right] r_{n}^{3}}{\left[1+2 \varepsilon_{h} / \varepsilon_{n}\right]\left[\varepsilon_{n}^{s}+2 \varepsilon_{n}\right] r_{n+1}^{3}+2\left[\varepsilon_{h}-\varepsilon_{n}+\varepsilon_{n}^{s}\left(1-\varepsilon_{h} / \varepsilon_{n}\right)\right] r_{n}^{3}} .
$$

In exactly the same way how the MLWA extends the result in Eq. (16) to that in Eq. (14), that in Eq. (17) can be extended in the same fashion leading to the following modified result: 


$$
\begin{gathered}
\frac{\left(1-\varepsilon_{h} / \varepsilon_{n}\right)\left[\chi_{n-1} \varepsilon_{n-1}^{s}+\left(1-\chi_{n-1}\right) \varepsilon_{n}\right] r_{n+1}^{3}+\left[\left(1-\chi_{n+1}\right)\left(\varepsilon_{n-1}^{s}-\varepsilon_{n}\right)+\chi_{n+1} \varepsilon_{h}\left(\varepsilon_{n-1}^{s} / \varepsilon_{n}-1\right)\right] r_{n-1}^{3}}{\left[\chi_{n+1}+\left(1-\chi_{n+1}\right) \varepsilon_{h} / \varepsilon_{n}\right]\left[\chi_{n-1} \varepsilon_{n-1}^{s}+\left(1-\chi_{n-1}\right) \varepsilon_{n}\right] r_{n+1}^{3}+\chi_{n+1}\left(1-\chi_{n+1}\right)\left[\varepsilon_{h}-\varepsilon_{n}+\varepsilon_{n-1}^{s}\left(1-\varepsilon_{h} / \varepsilon_{n}\right)\right] r_{n-1}^{3}} \\
=\frac{\left(1-\varepsilon_{h} / \varepsilon_{n}\right)\left[\chi_{n} \varepsilon_{n}^{s}+\left(1-\chi_{n}\right) \varepsilon_{n}\right] r_{n+1}^{3}+\left[\left(1-\chi_{n+1}\right)\left(\varepsilon_{n}^{s}-\varepsilon_{n}\right)+\chi_{n+1} \varepsilon_{h}\left(\varepsilon_{n}^{s} / \varepsilon_{n}-1\right)\right] r_{n}^{3}}{\left[\chi_{n+1}+\left(1-\chi_{n+1}\right) \varepsilon_{h} / \varepsilon_{n}\right]\left[\chi_{n} \varepsilon_{n}^{s}+\left(1-\chi_{n}\right) \varepsilon_{n}\right] r_{n+1}^{3}+\chi_{n+1}\left(1-\chi_{n+1}\right)\left[\varepsilon_{h}-\varepsilon_{n}+\varepsilon_{n}^{s}\left(1-\varepsilon_{h} / \varepsilon_{n}\right)\right] r_{n}^{3}} .
\end{gathered}
$$

The remaining steps for the derivation of the MLWA dipole polarizability for a $n$-layered nanomatryoshka are then very similar to those in Eqs. (6)-(10). Thus by defining

$$
H_{n}=\frac{1}{3} \frac{\left.\left(\varepsilon_{n}-\varepsilon_{h}\right)\left[\chi_{n-1} \varepsilon_{n-1}^{s}+\left(1-\chi_{n-1}\right) \varepsilon_{n}\right] r_{n+1}^{3}+\left(\varepsilon_{n-1}^{s}-\varepsilon_{n}\right)\left[\left(1-\chi_{n+1}\right) \varepsilon_{n}+\chi_{n+1} \varepsilon_{h}\right] r_{n-1}^{3} \varepsilon_{n-1}^{s}+\left(1-\chi_{n-1}\right) \varepsilon_{n}\right]\left[\chi_{n+1} \varepsilon_{n}+\left(1-\chi_{n+1}\right) \varepsilon_{h}\right] r_{n+1}^{3}+\chi_{n+1}\left(1-\chi_{n+1}\right)\left(\varepsilon_{n-1}^{s}-\varepsilon_{n}\right)\left(\varepsilon_{n}-\varepsilon_{h}\right) r_{n-1}^{3}}{,},
$$

one can solve from Eq. (18) and obtain $\varepsilon_{n}^{s}$ in the following form:

$$
\begin{gathered}
\varepsilon_{n}^{s}=\frac{1-\left(1 / \chi_{n}-1\right) p_{n}}{1+p_{n}} \varepsilon_{n}, \\
\text { where } \quad p_{n}=\frac{\chi_{n}\left(1-3 \chi_{n+1} H_{n}\right) \varepsilon_{n}-\chi_{n}\left[1+3\left(1-\chi_{n+1}\right) H_{n}\right] \varepsilon_{h}}{\left(1-\chi_{n+1}\right)\left(1-3 \chi_{n+1} H_{n}\right) \varepsilon_{n}+\chi_{n+1}\left[1+3\left(1-\chi_{n+1}\right) H_{n}\right] \varepsilon_{h}}\left(\frac{r_{n+1}}{r_{n}}\right)^{3} .
\end{gathered}
$$

Again, successive iteration with $\varepsilon_{1}^{s}=\varepsilon_{1}$ allows one to obtain finally the following MLWA dipole polarizability for the $n$-layered system in the form

$$
\alpha_{1}=r_{n+1}^{3} J_{n+1}
$$

where

$$
J_{n}=\frac{1}{3} \frac{\left(\varepsilon_{n}-\varepsilon_{h}\right)\left[\chi_{n-1} \varepsilon_{n-1}^{s}+\left(1-\chi_{n-1}\right) \varepsilon_{n}\right] r_{n}^{3}+\left[\left(1-\chi_{n}\right)\left(\varepsilon_{n-1}^{s}-\varepsilon_{n}\right) \varepsilon_{n}+\chi_{n} \varepsilon_{h}\left(\varepsilon_{n-1}^{s}-\varepsilon_{n}\right)\right] r_{n-1}^{3}}{\left.\left(1-\chi_{n}\right) \varepsilon_{h}\right]\left[\chi_{n-1} \varepsilon_{n-1}^{s}+\left(1-\chi_{n-1}\right) \varepsilon_{n}\right] r_{n}^{3}+\chi_{n}\left(1-\chi_{n}\right)\left(\varepsilon_{n-1}^{s}-\varepsilon_{n}\right)\left(\varepsilon_{n}-\varepsilon_{h}\right) r_{n-1}^{3}}
$$

\section{NUMERICAL RESULTS}

To demonstrate the nonlocal effects for the nanomatryoshka, we have considered the four-layer system first studied by Prodan et al. in Ref. 9, which can be described by setting $n=3$ in our above Eqs. (6)-(10). Here we shall assume the metal to be silver and insulator to be glass in a vacuum environment $\left(\varepsilon_{h}=1\right)$. The metal is described by a nonlocal hydrodynamic function as follows:

$$
\varepsilon(k, \omega)=1-\frac{\omega_{p}^{2}}{\omega(\omega+i \Gamma)-v_{0}^{2} k^{2}},
$$

for which the integral in Eq. (2) can be performed analytically and the result can be obtained in terms of the modified Bessel functions as follows: ${ }^{20,21}$

$$
\begin{aligned}
E_{\ell, n}^{p, q}= & {\left[\frac{1}{\varepsilon_{n}(\omega)}+(2 \ell+1)\left(\frac{r_{q}}{r_{p}}\right)^{\ell+1 / 2}\right.} \\
& \left.\times\left(\frac{\omega_{p}^{2}}{\omega_{p}^{2}-\omega(\omega+i \Gamma)}\right) I_{\ell+1 / 2}\left(\kappa r_{p}\right) K_{\ell+1 / 2}\left(\kappa r_{q}\right)\right]^{-1},
\end{aligned}
$$

where $\kappa=\sqrt{\omega_{p}^{2}-\omega(\omega+i \Gamma)} / v_{0}, v_{0}=\sqrt{3 / 5} v_{F}$ and we have assumed $r_{p}<r_{q}$. The damping constant including the interfacial scattering effects at a shell boundary can be obtained as $\Gamma=\Gamma_{B}+A v_{F} /\left(r_{q}-r_{p}\right)$ together with the following parameters for $\mathrm{Ag}:^{21} \omega_{p}=1.36 \times 10^{16} \mathrm{~s}^{-1}, \quad \Gamma_{B}=2.56 \times 10^{13} \mathrm{~s}^{-1}, \quad v_{F}$ $=1.39 \times 10^{15} \mathrm{~nm} / \mathrm{s}$, and we also set $A \approx 1$ for most our calculations. Note that strictly speaking this surface scattering term $(\sim A)$ has its own nonlocal characteristics which can invalidate the simple nonlocal dependence of the dielectric function in the form $\varepsilon\left(\vec{r}, \vec{r}^{\prime}\right)=\varepsilon\left(\left|\vec{r}-\vec{r}^{\prime}\right|\right)$, making a response of the type $\varepsilon(k, \omega)$ inapplicable. However this will go beyond our present limitation and we shall leave this for more exact treatment as a future challenge. Instead, we shall here make a first approximation assuming that such surface scattering can be treated in a similar way as in the local response case $(k$ $=0$ ) yielding simply a modified mean-free path for the free electrons in the metal. ${ }^{21}$ To illustrate the effects of this term, we have plotted in Fig. 3 the electric field intensity just outside a four-layer structure which is similar to that studied in Ref. 9 but with much smaller dimensions for which nonlocal effects are manifested. Thus we have two plasmonic shells of $\mathrm{Ag}$ interacting with each other in this structure: one at $1<r<2 \mathrm{~nm}$ and the other at $3<r<4 \mathrm{~nm}$, with the core and the spacer between them being glass, and we have calculated the field intensity at a point $1 \mathrm{~nm}$ outside the north pole of the multishell structure for both the cases $A=0$ and $A=1$, assuming a uniform incident field $E_{0}$ to be along the $z$ axis. From the results, one sees that the surface scattering leads to strong suppression of the surface fields, and it appears to affect to a somewhat less extent in the nonlocal case [Fig. 3(b)]. Furthermore, the bulk resonances for frequencies above that of the plasmon frequency in the nonlocal case are 


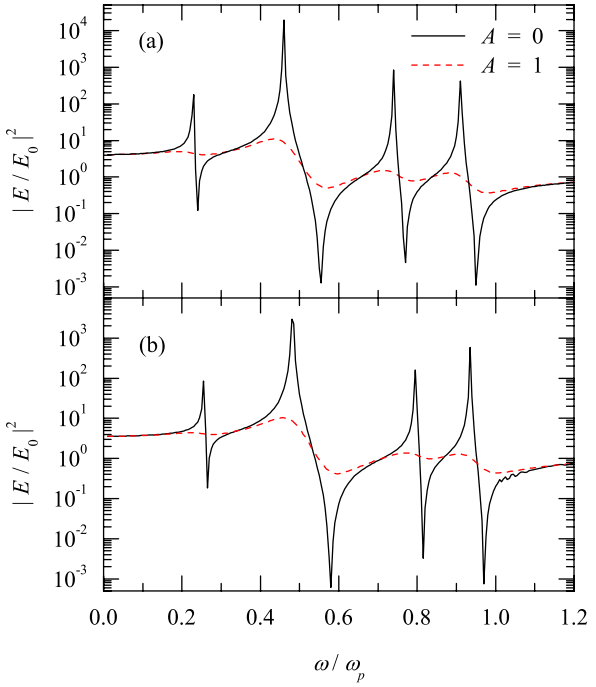

FIG. 3. (Color online) Intensity of the radial component of the electric field at a point $1 \mathrm{~nm}$ outside the north pole of a four-layer shell with radii $r_{1}=1 \mathrm{~nm}, r_{2}=2 \mathrm{~nm}, r_{3}=3 \mathrm{~nm}, r_{4}=4 \mathrm{~nm}$ for (a) local and (b) nonlocal models. The shell is made of (from core) glass/silver/glass/silver. Note the weak bulk plasmon resonances above $\omega_{p}$ excited in (b) in the absence of surface scattering.

all suppressed due to this surface damping effect.

We next consider the interaction of light with the same nanomatryoshka as in Fig. 3. Figure 4(a) shows the calculation of the extinction coefficients ${ }^{25}$ in which we show both the nonlocal calculations and the local results [by setting $k$ $=0$ in Eq. (24)] for comparison. First it is clearly seen that the dipolar plasmon of this nanomatryoshka is manifested in four coupled modes (consistent with those revealed in Fig. 3) as described by the hybridization model: ${ }^{9,11}$ two pairs of symmetric/antisymmetric modes centered around the high frequency antibonding mode $\left(\omega \sim 0.8 \omega_{p}\right)$ and the lowfrequency bonding mode $\left(\omega \sim 0.3 \omega_{p}\right)$, respectively. It is also noticeable that the strongest absorption mode for this structure occurs with the symmetric mode hybridized from the bonding modes of the shells. This happens since the induced dipole moments of all the "cavity modes" and "sphere modes" are enhancing with each other, resulting in a maximum induced dipole for the whole multishell system according to the hybridization model..$^{9,11}$ Such distinction between the symmetric and antisymmetric modes can also be seen from those hybridized at higher frequencies, though to a less extent. With the nonlocal effects of the metal accounted for, all the hybridized modes are slightly blueshifted as observed previously for simpler single-particle and single-shell systems. ${ }^{19-21}$ Furthermore, except for the strongest mode, the nonlocal effects lead to a slightly weaker peak for all the other absorption resonances. Such feature has not been observed previously with simpler particle or shell systems.

In Fig. 4(b), we have shown the results for a "complementary system" [with respect to the one in Fig. 4(a)] with the metal and dielectric layers "switched." Thus we have a Ag core for $r<1 \mathrm{~nm}$ and a Ag shell for $2<r<3 \mathrm{~nm}$ with only three interfacial plasmons interacting.

This results in three possibilities of coupling in the interfacial plasmons: (i) two antibonding modes with resonance

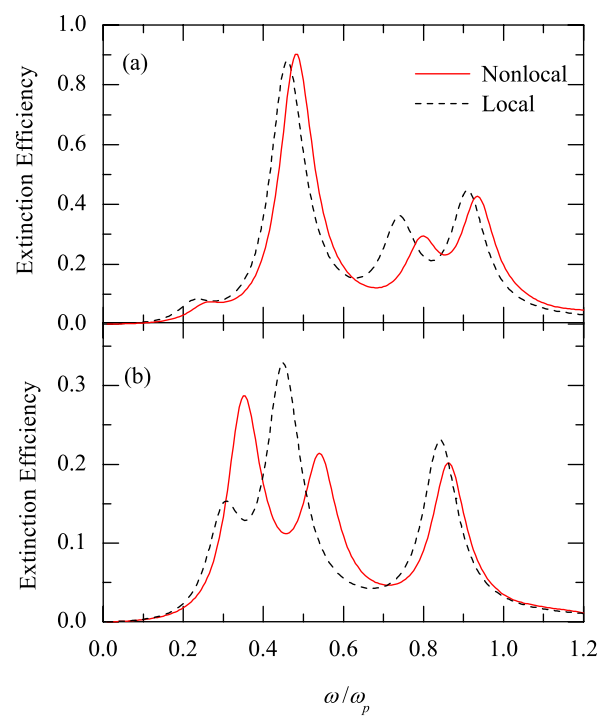

FIG. 4. (Color online) Extinction efficiency for a four-layer shell of the same dimensions as those in Fig. 3. The shell is made of (from core) glass/silver/glass/silver for case (a), and silver/glass/ silver/glass for case (b).

at $\omega \sim 0.8 \omega_{p}$, (ii) one antibonding and one bonding mode at $\omega \sim 0.5 \omega_{p}$, and (iii) two bonding modes at $\omega \sim 0.3 \omega_{p}$. It is interesting to note that the nonlocal effects have reversed the relative strength of the total induced dipole moment for the multishell system between the modes in (ii) and (iii), and have again led to blueshifts in the three resonance frequencies.

We next study the nonlocal effects on the nanomatryoshka in Fig. 3 as a function of the multipole order at fixed optical frequency, and the results are shown in Fig. 5. These results are qualitatively similar to those reported previously for the

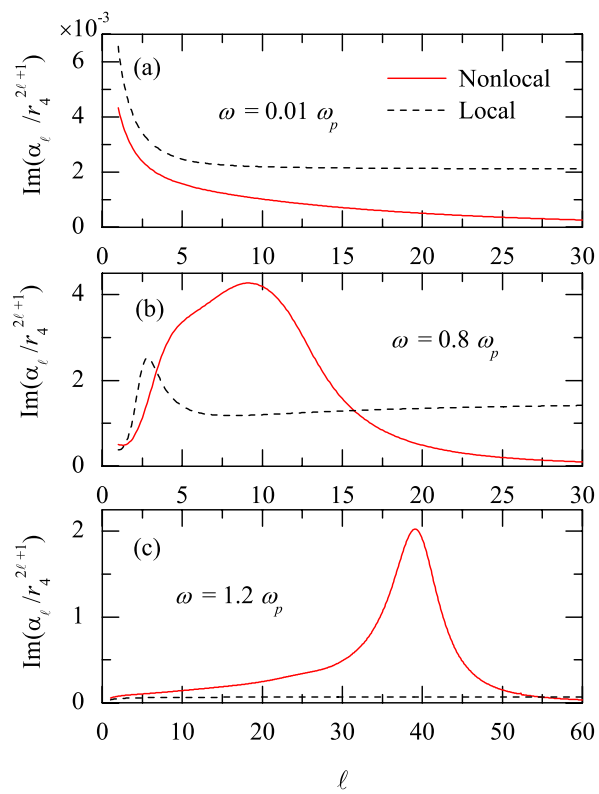

FIG. 5. (Color online) Imaginary part of the $\ell$-pole polarizability of the shell in Fig. 4(a) as a function of the pole order. The frequency $\omega$ is fixed at (a) $0.01 \omega_{p}$, (b) $0.8 \omega_{p}$, and (c) $1.2 \omega_{p}$, respectively. 


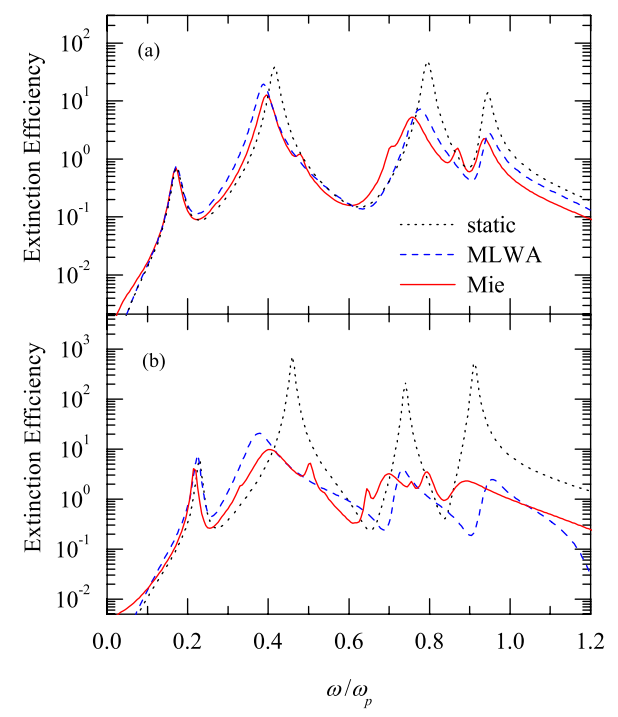

FIG. 6. (Color online) Comparison of the extinction efficiencies according to the LWA, MLWA, and the exact Mie results for a four-layer nanomatryoshka composed of: glass/Ag/glass/Ag of dimensions (a) $10 \mathrm{~nm} / 15 \mathrm{~nm} / 20 \mathrm{~nm} / 25 \mathrm{~nm}$, and (b) $10 \mathrm{~nm} / 20 \mathrm{~nm} / 30$ $\mathrm{nm} / 40 \mathrm{~nm}$, respectively.

case of a single nonlocal sphere ${ }^{19}$ and those for a single shell. ${ }^{20,21}$ At low frequencies, the nonlocal effects make the high-order contributions to the extinction coefficient much more insignificant than those in the local theory.

At higher frequencies, however, contributions from certain range of higher orders will become very prominent as can be seen in Figs. 5(b) and 5(c) since the nonlocal effects will have significant blueshifts for higher-order plasmon resonances. ${ }^{21}$ Thus higher-order contributions to the extinction of the nanomatryoshka will have to be included even for its small dimensions at high scattering frequencies, if nonlocal optical response of the nanoshells has to be accounted for.

We next study the effects of the MLWA on the dipole polarizability of the nanomatryoshka for shells of larger dimensions. Figure 6 shows the comparison of the results for extinction coefficients obtained for the "double-shell system" of not-too-large dimensions from the three theories: the quasistatic LWA, the MLWA, and the exact Mie scattering theory for multishells. ${ }^{26,27}$ We have calculated the exact Mie results by directly programming the theory given in Ref. 27 . As is clear from the results here for shells of dimensions (external radii) $\sim 25 \mathrm{~nm}$ and $40 \mathrm{~nm}$, respectively, the LWA results are not too bad, but progressively becoming inaccurate as the shell size increases. The MLWA and the Mie results, however, agree extremely well in both of these two cases considered.

Next we show in Fig. 7 similar results for two matryoshkas of greater dimensions: $60 \mathrm{~nm}$ and $80 \mathrm{~nm}$, respectively. It is noticeable that for systems of such dimensions not too small compared to the wavelength, the LWA totally fails except perhaps with limited validity only for the lowestfrequency resonance absorption of the system. On the other hand, the MLWA generally leads to (i) smaller extinction coefficients, (ii) redshifts in the dipole resonances, and most

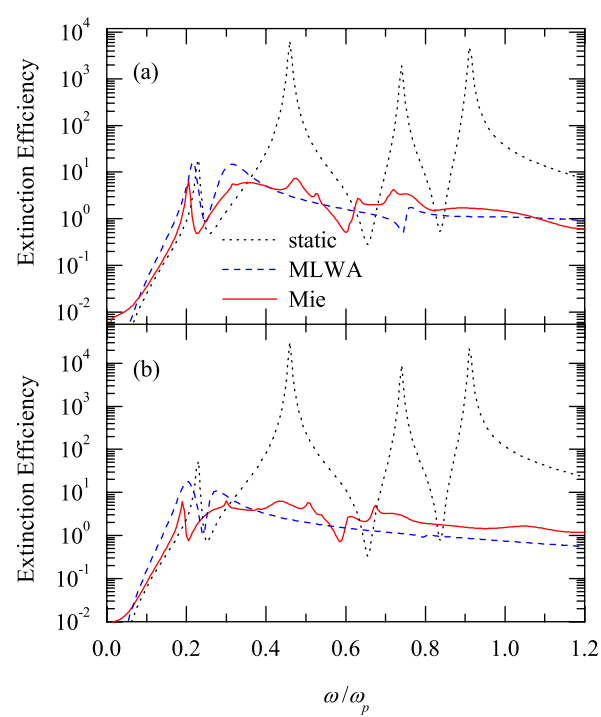

FIG. 7. (Color online) Same as in Fig. 6 but for two larger systems of dimensions (a) $15 \mathrm{~nm} / 30 \mathrm{~nm} / 45 \mathrm{~nm} / 60 \mathrm{~nm}$, and (b) 20 $\mathrm{nm} / 40 \mathrm{~nm} / 60 \mathrm{~nm} / 80 \mathrm{~nm}$, respectively.

interestingly, (iii) the disappearance in the high-frequency hybridized dipole resonances. The understanding of the results in (i) and (ii) can be referred to the effects from the dynamic polarization and the radiation loss, which lead clearly to a smaller polarizability and lower resonance frequency in the simple single-particle case. ${ }^{22-24}$ The manifestation of the observation in (iii), however, shows the significant breakdown of the static dipole approximation (on which the hybridization model is based) for these high-frequency modes where the long wavelength approximation is completely not justified except for systems of sufficiently small dimensions. The retardation effects simply make the highfrequency modes from the dipole hybridization of the interfacial plasmons disappear. In comparison with the exact Mie results, however, the MLWA still pertains to a certain degree of validity for matryoshkas of these rather large dimensions, but certainly starts to loss its accuracy, especially for the high-frequency resonances for the $80 \mathrm{~nm}$ system.

\section{DISCUSSION AND CONCLUSION}

In the theoretical understanding of various optical phenomena in nanoplasmonics, the long wavelength approximation has proven to be a simple and useful tool, especially in dealing with the optical interaction with MNPs. However, for dimensions which are either ultrasmall $(<10 \mathrm{~nm})$ or nottoo-small $(\sim 100 \mathrm{~nm})$, significant modifications are necessary to achieve sufficient accuracy in the optical characterization of these MNPs using quasistatic theories. We have considered here the nonlocal optical effects and the finitewavelength effects in each of the two limits of particle sizes for the nanomatryoshka.

Thus we have in this work showed that it is possible to extend the applicability of the simple long wavelength quasistatic description of the optical response of the nanomatryoshka to dimensions from ultrasmall ones to those up to 
$\sim 10 \%$ of the wavelengths. Recent experiments have revealed blueshifts in the plasmonic resonance of ultrasmall gold particles, ${ }^{28}$ as well as the significance of nonlocal effects in aggregates of MNPs. ${ }^{29}$ Our models so derived here should be useful when similar experiments are performed with the nanomatryoshka of such small dimensions. In addition, the MLWA we formulated for the nanomatryoshka should enable one to perform an accurate quasistatic analysis in the optical characterization of these particles of dimensions up to $\sim$ tens of nanometers. In spite of the fact that fully dynamic formulation in terms of the transfer matrices has been available in the literature for the nanomatryoshka, ${ }^{10}$ we believe our present work is of value for the simplicity of the long wavelength approximation (e.g., without using complicated vector harmonics), and its sufficient accuracy after incorporation of the effects introduced here, over a significant range of shell sizes often encountered in various optical experiments. In addition, the distinct higher multipole results which can be obtained rather straightforwardly from the present formulation will be useful for the description of nearfield interactions with these structures. ${ }^{21,30}$

A challenge remains in our formulation which is to merge the two effects to account for the nonlocal dielectric response of the metallic shells in the MLWA for the nanomatryoshka. This will be necessary, for example, when one experiments with such systems of not-too-small sizes which may contain ultrathin metal shells "sandwiched" between dielectric shells of relatively thick dimensions. However, we have encountered difficulties in our present formulation of the nonlocal polarizability for the nanomatryoshka which can at the most reduce a multishell system to an effective single-shell structure but not to an effective solid sphere as is possible with the nanomatryoshka limiting only to local dielectric response. ${ }^{13}$ Since the conventional approach in the MLWA has to refer to only a solid sphere but not a shell-like structure, some innovative ideas are necessary to combine the two effects we studied in this paper in order to formulate a nonlocal MLWA for the description of the optical properties of the metallic nanomatryoshka. In addition, the MLWA formulated thus far is limited only to dipole response and the generalization of it to higher multipoles is not trivial. We hope to revisit these issues in a future endeavor.

\section{ACKNOWLEDGMENTS}

We thank Alexander Moroz for his correspondence, and Huai-Yi Xie for discussion. This research is supported by the National Center for Theoretical Sciences of Taiwan and the Center for Marine Bioenvironment and Biotechnology at National Taiwan Ocean University. We also acknowledge the support of the National Science Council of Taiwan under Grants No. NSC 97-2112-M-019-001-MY3 and No. NSC99-2120-M-002-012.
*Corresponding author.

†b89202048@ntu.edu.tw

*hopl@pdx.edu

${ }^{1}$ E. Ozbay, Science 311, 189 (2006).

${ }^{2}$ See, e.g., Advances in Metal-Molecular Interactions, edited by C. D. Geddes and M. H. Chowdhury, special issue of Plasmonics 2(3) (2007).

${ }^{3}$ For a recent review, see, e.g., S. A. Maier and H. A. Atwater, J. Appl. Phys. 98, 011101 (2005).

${ }^{4}$ K. L. Kelly, E. Coronado, L. L. Zhao, and G. C. Schatz, J. Phys. Chem. B 107, 668 (2003).

${ }^{5}$ S. J. Oldenburg, R. D. Averitt, S. L. Westcott, and N. J. Halas, Chem. Phys. Lett. 288, 243 (1998).

${ }^{6}$ L. R. Hirsch, A. M. Gabin, A. R. Lowery, F. Tam, R. A. Drezek, N. J. Halas, and J. L. West, Annals Biomed. Engineering 34, 15 (2006).

${ }^{7}$ H. Wang, D. W. Brandl, F. Le, P. Nordlander, and N. J. Halas, Nano Lett. 6, 827 (2006).

${ }^{8}$ A. Steinbrück, A. Csáki, G. Festag, and W. Fritzsche, Plasmonics 1, 79 (2006).

${ }^{9}$ E. Prodan, C. Radloff, N. J. Halas, and P. Nordlander, Science 302, 419 (2003).

${ }^{10}$ A. Moroz, Ann. Phys. (N.Y.) 315, 352 (2005); Chem. Phys. 317, 1 (2005).

${ }^{11}$ E. Prodan and P. Nordlander, J. Chem. Phys. 120, 5444 (2004); see also J. M. Steele et al., in Surface Plasmon Nanophotonics, edited by M. L. Brongersma and P. G. Kik, Springer Series in Optical Sciences Vol. 131 (Springer, Dordrecht, 2007), pp. 183196.
${ }^{12}$ N. V. Voshchinnikov and J. S. Mathis, Astrophys. J. 526, 257 (1999); J. Li, G. Sun, and C. T. Chan, Phys. Rev. B 73, 075117 (2006).

${ }^{13}$ H. Y. Chung, P. T. Leung, and D. P. Tsai, J. Chem. Phys. 131, 124122 (2009).

${ }^{14}$ W. Ekardt, Phys. Rev. B 31, 6360 (1985); 34, 526 (1986).

${ }^{15} \mathrm{~K}$. Cho, Optical Response of Nanostructures (Springer-Verlag, Berlin, 2003).

${ }^{16}$ J. M. McMahon, S. K. Gray, and G. C. Schatz, Phys. Rev. Lett. 103, 097403 (2009).

${ }^{17}$ J. M. McMahon, S. K. Gray, and G. C. Schatz, Phys. Rev. B 82, 035423 (2010).

${ }^{18}$ J. M. McMahon, S. K. Gray, and G. C. Schatz, Nano Lett. 10, 3473 (2010).

${ }^{19}$ R. Fuchs and F. Claro, Phys. Rev. B 35, 3722 (1987).

${ }^{20}$ R. Rojas, F. Claro, and R. Fuchs, Phys. Rev. B 37, 6799 (1988).

${ }^{21}$ R. Chang and P. T. Leung, Phys. Rev. B 73, 125438 (2006); 75, 079901(E) (2007).

${ }^{22}$ M. Meier and A. Wokaun, Opt. Lett. 8, 581 (1983).

${ }^{23}$ A. Moroz, J. Opt. Soc. Am. B 26, 517 (2009).

${ }^{24}$ H. Mertens, A. F. Koenderink, and A. Polman, Phys. Rev. B 76, 115123 (2007).

${ }^{25}$ The extinction coefficient is defined as the sum of the absorption and scattering efficiencies: $Q_{a b}+Q_{s c}$, where $Q_{a b}=\frac{8 \pi}{\lambda} \operatorname{Im}\left(\frac{\alpha_{1}}{r_{m}^{2}}\right)$ and $Q_{s c}=\frac{8}{3}\left(\frac{2 \pi}{\lambda}\right)^{4}\left|\frac{\alpha_{1}}{r_{m}}\right|^{2}$, with $r_{m}$ being the outermost radius of the multishell structure.

${ }^{26}$ R. Bhandari, Appl. Opt. 24, 1960 (1985); Z. S. Wu and Y. P. Wang, Radio Sci. 26, 1393 (1991); B. R. Johnson, Appl. Opt. 
35, 3286 (1996).

${ }^{27}$ W. Yang, Appl. Opt. 42, 1710 (2003).

${ }^{28}$ S. Palomba, L. Novotny, and R. E. Palmer, Opt. Commun. 281, 480 (2008).
${ }^{29}$ A. Pack, M. Hietschold, and R. Wannemacher, Opt. Commun. 194, 277 (2001).

${ }^{30}$ H. Y. Xie, H. Y. Chung, P. T. Leung, and D. P. Tsai, Phys. Rev. B 80, 155448 (2009). 